\title{
ECONOMICS AND EVOLUTION
}

VERMEIJ*, Geerat J., Department of Geology, University of California at Davis, Davis, CA 95616 U. S. A.

Individual organisms compete for resources. Among competitive dominants, per-capita energy use has generally increased through time. This increase has had a ripple effect on all other species by increasing the number of competitive and predatory encounters among individuals. Species unable to cope with such biological rigors have become restricted to environments where resource supply is low and where encounters with enemies are few. Among species that hold their own in biologically rigorous habitats, construction materials that are cheap to produce and that enable individuals to grow and respond quickly have generally been favored over those that exact a high cost in energy and time. Extinction interrupts but does not reverse or fundamentally alter these long-term between-clade evolutionary trends. The availability of resources to organisms, as well as the opportunity for evolutionary change, depends on extrinsic events and factors as well as on the competitive abilities of organisms.

Those who have raised methodological and theoretical objections against this economic interpretation of the history of life deny the overriding importance of organisms as agents of natural selection, emphasize the random nature of extinction, deny the existence of long-term trends, favor a larger role for mutualistic as opposed to antagonistic interactions, or accord a larger role to species-level attributes in evolution that are not reducible to the properties of individual organisms. These arguments are either unpersuasive or incorrect. The long-term economics of life may have important lessons for our own use of resources. 